\title{
Die untere Grenze von Strukturbodenformen in den Gurktaler und Seetaler Alpen
}

\author{
Von Gudrun HöHL, Bamberg \\ Mit 4 Abb. im Text
}

\begin{abstract}
$\mathrm{Zus}$ a m menfassung. Auf Grund der im Untersuchungsbereiche bis $1500 \mathrm{~m}$ herab verfolgten, zahlreichen Miniatursteinringe wird die Frage aufgeworfen, ob die für den randalpinen Raum in $1800-2000 \mathrm{~m}$ Höhe angenommene "klimatische Strukturbodengrenze" nicht noch eine unterste Zone, nämlich die der Initialformen, einschließen müßte, um wirklich Untergrenze $\mathrm{zu}$ sein.

$\mathrm{Su} \mathrm{m} \mathrm{m}$ a r y. On account of numerous miniature stone networks observed in the examined region down to 1500 metres, the question is raised, whether the "border-line of climatic patterned ground" - which is supposed to run in an altitude of 1800 or 2000 metres in the zone around the Central Alpes - should not include another zone (of the „initial forms“) below the above mentioned line. And the question arises: isn't this the real lower border-line?
\end{abstract}

Anläßlich meiner Beobachtungen über Doppelgrate in den nordöstlichen Gurktaler Alpen im Jahre 1950 (G. HöHL 1953) wurde ich auf Miniaturstrukturbodenformen (Steinringe und Steinnetzwerke) aufmerksam. Da die Vorkommen in tieferer Lage festgestellt werden konnten als bisher in der Literatur angegeben, wurden die früheren Begehungen und Aufnahmen im Jahre 1953 fortgesetzt. Als Arbeitsgebiet wurden wiederum die Gurktaler Alpen, diesmal im Bereich der Turracher Höhe, gewählt, wie auch der Nordwesten der Seetaler Alpen. Wenn die Beobachtungen auch nur Ausschnitte aus dieser Landschaft berücksichtigen konnten, so sind sie doch geeignet, ergänzende Bemerkungen über die Verbreitung von Strukturbodenformen am Rande des zentralalpinen Raumes zu machen.

Bisherige Beobachtungen von Strukturbodenformen in den Ostalpen (Chr. TARnuzzer 1909 u. 1911, H. Waldbaur 1921, N. Krebs 1925 u. 1928, H. KINZL 1928, W. Salomon 1929, W. Mohaupt 1932, F. Mattick 1941, C. Troll 1944, G. Eberle 1952) brachten jeweils Beispiele aus Regionen von durchschnittlich $2000 \mathrm{~m}$ an aufwärts ${ }^{1}$ ). Auf Grund dieser Vorkommen konnte eine untere Grenze der Verbreitung von frostbedingten Materialsortierungen festgestellt werden (H. Poser 1933, J. BüDEL 1937 u. 1944), die Troll (1948, S. 5; vgl. auch 1947, S. 163 f. u. 1944, S. 554) „klimatische Strukturbodengrenze" genannt und für den zentralalpinen Raum in einer Höhe von $2000-2200 \mathrm{~m}$, für den randalpinen Raum bei $1800-2000 \mathrm{~m}$ festgelegt hat. Oberhalb dieser sog. klimatischen Strukturbodengrenze ergab sich infolge verschiedener Entstehungsbedingungen und Ausbildungsweise der Strukturformen die Möglichkeit zu deren zonaler Gliederung, indem in einem unteren Gürtel von ca. 2000_-2600 m Höhe größere, an starke Wasserdurchtränkung in dem Vorgelände rezenter Gletscher gebundene Steinringe vorherrschen, die dem jahreszeitlichen polaren Typus entsprechen, während in einem oberen Gürtel von ca. $2700 \mathrm{~m}$ an zur Hauptsache ausgesprochene Miniaturformen vorkommen, die dem durch den tageszeitlichen Frostwechselrhythmus bedingten tropischen Typus vergleichbar sind (Troll 1944, S. 655 u. 677; 1947, S. 165). U n t e r h a lb der "klimatischen Strukturbodengrenze" ganz vereinzelt beobachtete Vorkommen waren durchwegs durch Wasserbedeckung, Höhlenklima oder Gesteinsart begünstigt

1) Vorkommen von Struktur- bzw. Streifenboden beobachtet von TARnUzzer in 2670 bis 2900 m, Unterengadin; Götzinger (1913) in $1850 \mathrm{~m}$, Schneealpe; BAEDEKER (1922) in $1850 \mathrm{~m}$, Schneealpe; KINZL in 2200-2600 m, Silvretta, Ótztaler u. Stubaier Alpen, Hohe Tauern mit Venediger Gruppe; KREBS in $2000-2500 \mathrm{~m}$, vor allem Schieferalpen; SALOMON in 2600-3000 m, Unterengadin; Mohaupt in 2300-2800 m, Stubai u. Dolomiten; MatTick in 2100-2200 m, Zillertaler u. Stubaier Alpen; EBERLE in $2100 \mathrm{~m}$, Allgäuer Alpen. 
(J. Schadler 1931, V. Conrad 1933, K. Leuchs 1933, Mohaupt 1932, Mattick 1941), so daß sie als „extrazonale“ bzw. „azonale" Strukturbodenvorkommen gesondert gestellt wurden (Troll 1944, S. 596 f., 1947, S. 163, 1948, S. 105).

Bei meinen Beobachtungen handelt es sich um Miniatursteinringe und -steinnetzwerke, die in einer Region von $2200-1500 \mathrm{~m}$ herab verfolgt wurden, um für die tiefer gelegenen Materialsortierungen Vergleichsmaterial mit den Formen an und über der "klimatischen Strukturbodengrenze“ am Rande der Alpen zu bekommen. Diese tiefgelegenen Frostbodenformen dürfen aber $\mathrm{n}$ ich $\mathrm{t}$ als „extrazonale“ Vorkommen gedeutet werden, was hier dargelegt werden soll. Sie haben neben den beiden Merkmalen einer Größe von 2-10 cm Durchmesser und der Höhenlage über und unter der „klimatischen Strukturbodengrenze" als weiteres Kennzeichen die Lage außerhalb der Gletschervorfelder (T'ROLL 1947, S. 652) im Gegensatz zu den von KINZL und EberLe beschriebenen Vorkommen, aber nicht die Vorzüge aklimatischer Begünstigung. Sie sind jedoch insofern zum Teil durch außergewöhnliche Umstände beeinflußt, als sie nicht nur auf den kleinen Schuttebenheiten (den Schneeterrassen), in der Hangschuttdecke, an flacheren Schutthängen in der Umgebung großer Schneeflecke, sondern auch auf den allenthalben vorhandenen Viehtrittpfaden, auf - bis zum Zeitpunkt meiner Begehung Ende Mai - unbegangenen Almwegen, im größeren Umkreis von Almbrunnen und an den durch Straßenbau angeschnittenen bergseitigen Hängen vorkommen. Beobachtet aber wurden sie nicht in direktem Zusammenhang mit den großartig ausgebildeten Erdbülten, wie sie vor allem am Aufstieg zur Wenzelalpe von Neumarkt aus in den Seetaler Alpen auftreten. Darüber und darunter jedoch konnten die Steinringe wieder verfolgt werden. Wo auch immer durch natürliche oder auch künstliche Voraussetzungen vegetationsfreies Schuttmaterial zur Verfügung steht, unterliegt es durchwegs den Frosteinwirkungen (vgl. BüDEL 1937). Daraus ergibt sich auf Schritt und Tritt ein Vorhandensein von Frostbodenformen als Ausdruck von Bodenbewegungen bis in die Waldzone hinein.

Daß der Frost auch in tiefen Lagen bis Ende Mai wirksam ist, konnte an Ort und Stelle im Gebiet der Turracher Höhe beobachtet werden, da noch in der Nacht vom 28. auf 29. Mai starker Frost, mit Neuschnee oberhalb $2000 \mathrm{~m}$, am Ende einer warmen Schönwetterperiode auftrat, durch welchen noch bis in die späten Vormittagsstunden die Lockererde bis $1500 \mathrm{~m}$ herunter hochgefroren und die Steinchen hochkant gestellt waren. Wie weit in das Jahr hinein Frostnächte im Gebiet der Gurktaler Alpen und auch Seetaler Alpen auftreten bzw. schon wieder beginnen, läßt sich zum Teil aus den klimatischen Daten für Österreich (R. KLein 1909, V. Conrad 1913, V. Paschinger 1937, S. 93 f.) entnehmen, wonach das in $1264 \mathrm{~m}$ Höhe gelegene Turrach nördlich der Turracher Höhe (1763 m) im März 27,0, im April 13,6 und noch im Mai 2,7 Frosttage hat und im September die Fröste schon wieder beginnen. Weiterhin weist der südlich der Turracher Höhe gelegene Ort Ebene Reichenau bei $1059 \mathrm{~m}$ Meereshöhe im März -12,1 $1^{\circ}$, im April $-3,5^{\circ}$ und im Mai noch $-0,6^{\circ}$ als mittlere Minima auf, die dann erst im Oktober wieder Minuswerte erlangen. Die Station St. Lambrecht in den nordöstlichen Gurktaler Alpen hat bei einer Höhenlage von $1036 \mathrm{~m}$ im März 22,8, im April 6,8 und im Mai noch 0,7 Frosttage zu verzeichnen, die im September wieder einsetzen. Außerste Frostgrenzen sind für Turrach der 29. Mai und der 20. Juli, für Ebene Reichenau Mai und Oktober und für St. Lambrecht der 18. Mai und der 17. September. Für die nördlichen Seetaler Alpen könnten nur die Stationen Neumarkt/Steiermark, Hüttenberg und Judenburg herangezogen werden, die für die entsprechenden Monate ähnliche Werte erkennen lassen. Noch wichtiger als Frosttage festzustellen, wäre es, die Frostwechseltage bzw. die Frostwechselhäufigkeit, die für die Bildung von Miniatursteinnetzen Bedingung sind (T ROLL 1943, S. 161 ff.; vgl. auch 1941), bei einer. Beurteilung der klimatischen Verhältnisse zu Grunde zu legen. Einen Anhaltspunkt 
hierfür bietet aber die Arbeit von SchröDER (1912). Nach ihm liegt die Maximalzone der Frostwechselhäufigkeit in den Alpen im Mittel bei $1410 \mathrm{~m}$ Höhe, und die Häufigkeit der Schwankungen um den Frostpunkt ist im nördlichen Teil meines Untersuchungsgebietes mit 121-160 Schwankungen, im südlichen Teil mit 81-120 Schwankungen angegeben.

Frühjahr und auch Herbst scheinen also auch hier die wichtige Zeit für die Entstehung der Frostbodenformen zu sein. Darauf deuten zugleich die Vorkommen von Steinringen auf den Almwegen in der Region um $1700 \mathrm{~m}$ im Gebiete der Turracher Höhe hin. Von dieser Höhenlage an aufwärts waren die Wege seit dem Herbst nicht wieder begangen. Die Häufigkeit der Steinringe und deren dem tropischen Typus entsprechende Größe von höchstens $10 \mathrm{~cm}$ Durchmesser mögen beweisen, daß sie wahrscheinlich der Frühjahrszeit eines Jahres ihre Entstehung verdanken und daß die Bedingungen hierzu durch klimatische Einflüsse und Gesteinsmaterial (vor allem Schiefer in Lockererde) sehr günstig sind. Es handelt sich demnach um rezente, z. T. sogar äußerst kurzfristige Bildungen von Miniatursteinringen. Die Seetaler Alpen waren im Bereich der Wenzelalpe - im Mai bereits zu sehr begangen, als daß man noch derartige, auf den Wegen als den vegetationsfreien Stellen vorkommende Materialsortierungen in besonders tiefen Lagen hätte feststellen dürfen. Daß dort aber die gleichen tiefen Vorkommen zu beobachten wären, ist nicht zu bezweifeln. Das Gebiet der nordöstlichen Gurktaler Alpen mit Kuhalpe $(1784 \mathrm{~m})$, Frauenalpe $(2004 \mathrm{~m})$ und Ackerlhöhe $(2044 \mathrm{~m})$ wurde anfangs September besucht, so daß keine Aussagen über gleichartige Vorkommen gemacht werden können, obwohl sicher auch hier keine Ausnahme besteht.

Weiterhin fällt auf, daß nicht eventuell eine Abstufung in der Größenordnung bei den Strukturbodenformen von der höheren zur tieferen Lage hin besteht. So, wie es größere Formen von $6-9 \mathrm{~cm}$ innerem Durchmesser in $1950 \mathrm{~m}$ Höhe gibt, so sind sie auch bei 1740 und $1700 \mathrm{~m}$ vorhanden, und ebenso treten Kleinstformen sowohl in den tieferen Lagen als auch in den höheren, hier des öfteren größeren Formen untergeordnet, auf. Es ist daher in dieser Weise kein Nachlassen des klimatischen Faktors zu bemerken, was auch durch die Klarheit der Formen unterstrichen wird. Ebenso können die Materialsortierungen in Einzelformen, wie auch in Vergesellschaftung auftreten, ungeachtet der Höhenlage, wenn auch über $2000 \mathrm{~m}$ Höhe Frostmusterböden größeren Ausmaßes häufiger zu beobachten sind. Aber selbst die Vorkommen zwischen 1500 und $1650 \mathrm{~m}$ zeigen eine durchaus klare Anordnung von Miniatursteinnetzwerken. Andererseits sind die Schneeterrassen und die sonst von der Vegetation freigelassenen Stellen in der Krummholz- und Almregion sehr oft so klein, daß sich auf ihnen nur eine Einzelform entwickeln kann. Das Gleiche ist in der Waldregion, z. B. am Schoberriegl und am Schöneben Nock, zu beobachten.

Im folgenden seien als Belege für die bisherigen Ausführungen die einzelnen Beobachtungsergebnisse, unterstützt von einigen Bildern aus dem reichen Aufnahmematerial, gebracht. Die Gurktaler und Seetaler Alpen, die sich aus mehreren, durch weite getragene Berghöhensysteme und Rückenniveaus gegliederten Höhenlandschaften mit dem Charakter eines glazial geformten Mittelgebirges (vgl. H. Spreitzer 1951, 1951a, PASCHINGER 1937) zusammensetzen, ragen über die Waldgrenze, die im Mittel bei $1800 \mathrm{~m}$ verläuft, auf. Dadurch sind die Voraussetzungen für die klimatische Höhenlage von frostbedingten Materialsortierungen gegeben, die ihrerseits in den verwitterten altkristallinen Schiefern, Phylliten und Gneisen der Hangschuttdecke eine weitere Begünstigung erfahren. Diese genannten Verhältnisse liegen auch in dem ersten Beobachtungsgebiet vor, den nordöstlichen Gurktaler Alpen. Auf der $1784 \mathrm{~m}$ Höhe erreichenden Kuhalpe, die unter dem Einfluß des Südwindes ab $1620 \mathrm{~m}$ ein Auflockern und allmähliches Zurückbleiben des Waldes zeigt, finden sich in der abgeflachten Gipfelregion zahl- 
reiche Miniatursteinringe, zu ganzen Steinnetzwerken zusammengeschlossen. Sie treten auf all den kleinen, ebenen Schneeterrassen der Rückenhöhe und der Abhänge bis mehrere Meter unter dem Gipfel auf. Das Gesteinsmaterial setzt sich aus Quarzphyllit- und Diabasschutt zusammen. Der Durchmesser dieser kleinen einfachen Strukturformen beträgt $5-10 \mathrm{~cm}$. Das Steinnetzwerk ist auf die obersten $1-2 \mathrm{~cm}$ der Bodenzone beschränkt, unter welchen sich dann $5-10 \mathrm{~cm}$ durch Frosthub völlig steinfreier Boden befindet. Nur unter den Ringen reichen die Steine etwas tiefer in den Boden hinein, wobei sie gelegentlich auch steilgestellt sind. Die Entstehung des Miniatursteinnetzwerkes ist ausschließlich an die Frühjahrsfröste gebunden. Auf der nordwestlich der Kuhalpe gelegenen Frauenalpe waren bis zu $1820 \mathrm{~m}$ Höhe infolge der dichten Rasendecke und der starken Begehung dieses Gebietes keinerlei Strukturbodenformen zu erkennen. Unmittelbar am Anstieg zum höheren Gelände im Bereich des 1800-m-Niveaus dagegen sind in den plattigen Schiefern bläulicher Färbung recht schöne Steinringe der gleichen Art und Größe wie an der Kuhalpe zu sehen. Es handelt sich hier um einen Tonschieferzug im Metadiabas. Über dieser bereits über der Waldgrenze befindlichen Region sind im Umkreis der Kuppe und an der Kuppe der Frauenalpe selbst (ab 1990 m) wiederum Strukturböden der gleichen Art zu beobachten, angelegt im Lockermaterial des Metadiabases. Auch auf der südlich anschließenden Ackerlhöhe sind in gleicher Höhenlage kleine Strukturbodenformen zu beobachten.

Bei der Begehung der Westhänge der nördlichen Seetaler Alpen von dem Rücken Perchauer Alm-Oberbergalm-Wenzelalpe $(2153 \mathrm{~m})$ und südlich des Grotscher Kars bis zur Kulmer Alpe konnten von $1800 \mathrm{~m}$ an im Bereich der Krummholz- und Mattenregion Miniatursteinringe festgestellt werden. Auch hier fanden sich diese Strukturformen auf den kleinen Ebenheiten der Schneeterrassen, mit einem inneren Durchmesser von $3-7 \mathrm{~cm}$ und mit $7-10 \mathrm{~cm}$ tiefer Lockererde im Kreisinneren. Unter dem Steinring war das Erdreich dichter von Steinen durchsetzt. Gesteinsmäßig handelt es sich hier um verwitterten Glimmerschiefer, Gneis und Amphibolit. Auch hier könnten die sehr häufigen, zugleich aber stark isolierten Vorkommen durch zahlreiche Abbildungen belegt werden.

Im Gebiet der Turracher Höhe führte die eine Begehung entlang der Westseite des Schoberriegls $(2204 \mathrm{~m})$ und des Schöneben Nocks $(1996 \mathrm{~m})$. Noch in der Lärchenwaldregion, von Fichten und Zirbelkiefern durchsetzt, konnte auch hier stets Miniatursteinnetzwerk beobachtet werden. Es bestand vorwiegend aus bläulich grauem, blättrigem Tonschiefer, dessen flache Verwitterungsstückchen häufig Senkrechtstellung zeigten. Fundstellen waren in diesem Bereich die größeren oder kleineren Schuttflächen nahe den Wassertrögen der Almen im Umkreis des Grünsees. Sie boten aber keine Anhalts-punkte zur Annahme stärkerer Durchfeuchtung, wohl infolge der leicht erhöhten Lage yegenüber der Umgebung, und damit allem Anschein nach keine aklimatische Begünstigung des Sortierungsvorganges. Da die Almen Ende Mai noch nicht wieder bewirtschaftet waren und auch noch kein Viehauftrieb stattgefunden hatte, waren die Strukturbodenbildungen dieses Frühjahrs, die fast nur senkrecht gestellte Steinchen aufwiesen, noch unzerstört. Die Größenverhältnisse schwankten um $5 \mathrm{~cm}$ Durchmesser. Die Höhenlage dieser Vorkommen betrug ca. $1775 \mathrm{~m}$. In $1750 \mathrm{~m}$ oberhalb des Grünsees fiel in der Nähe eines mächtigen Schuttstromes ein Steinnetzwerk auf einer kleinen Schneeterrasse auf, dessen knapp $3 \mathrm{~cm}$ im Durchmesser großer Hauptsteinring einen $2 \mathrm{~cm}$ hoch gehobenen Feinerdekern zeigte. Ununterbrochen konnten dann Steinnetzwerke auf den Almwegen am Westhang des Schoberriegls und des Schöneben Nocks festgestellt werden. Sehr schöne Materialsortierung von größeren Steinen bis zur Feinerde ist auf Abb. $1 \mathrm{zu}$ erkennen. Die Höhenlage des ca. $6 \mathrm{~cm}$ im Durchmesser großen Steinringes (Pfeil 3), um welchen sich mehrere, etwas kleinere Ringe scharen (Pfeile 1, 2 und 4 bis 7), war in $1740 \mathrm{~m}$ am Schoberriegl. Auch am Westhang des Schöneben Nocks waren 


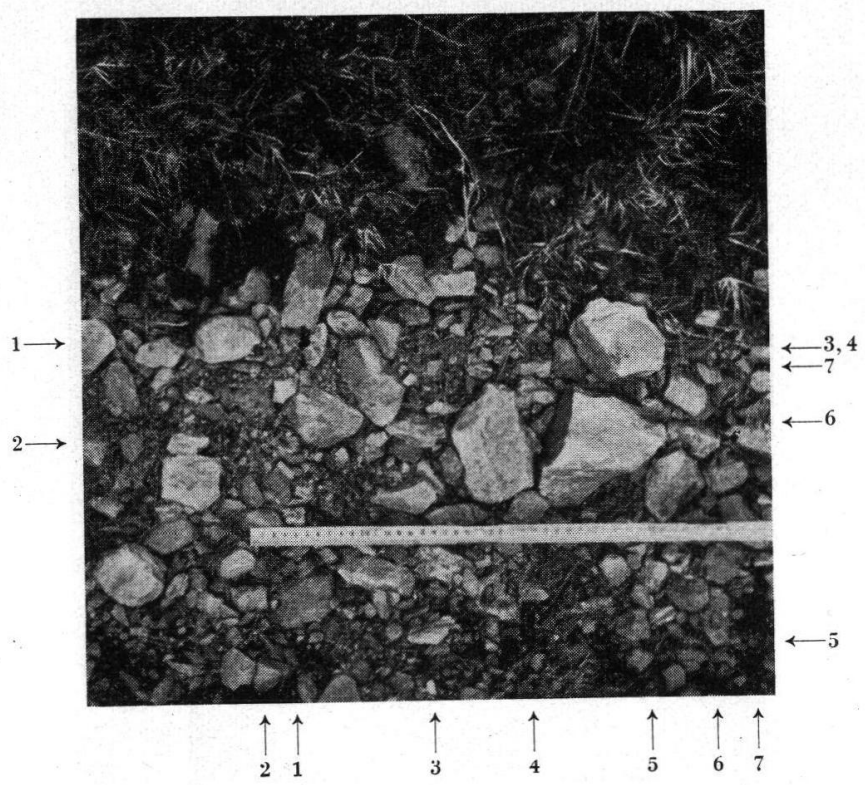

Abb. 1. Steinnetzwerk in $1740 \mathrm{~m}$ Höhe am Westhang des Schoberriegls/Kärnten. Materialsortierung in Grünschiefer auf einem Almweg südl. des Grünsees. Lärchenwaldregion. - Aufn. 27. 5. 1953.

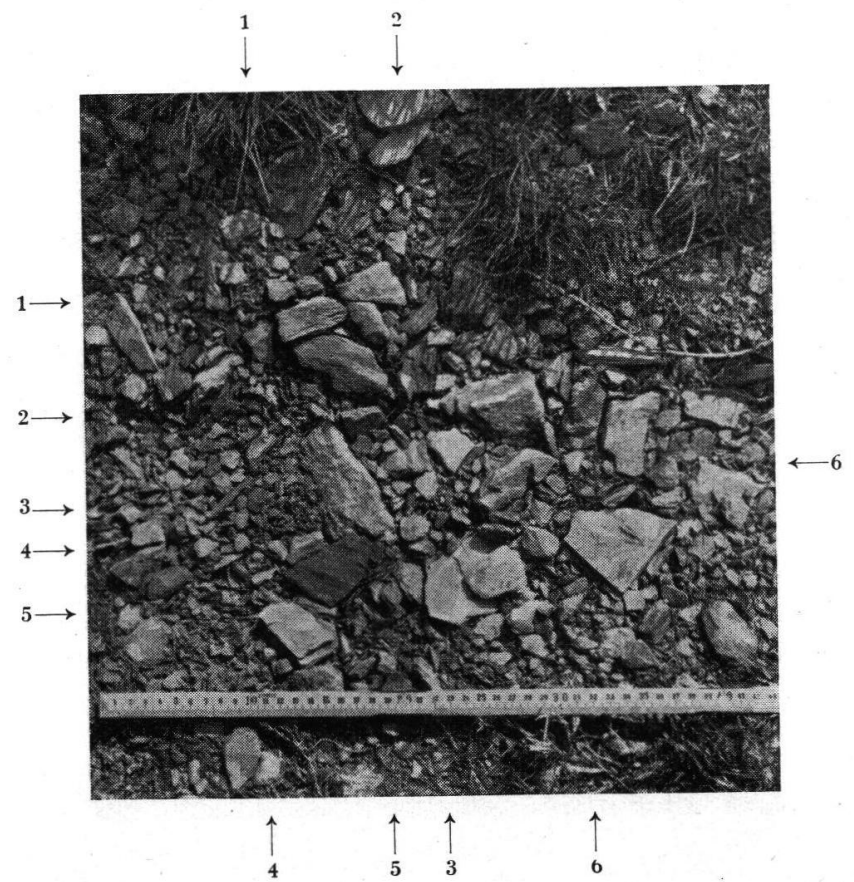

Abb. 2. Steinnetzwerk in $1700 \mathrm{~m}$ Höhe auf dem Almweg zur Natzalm am Westhang des Schơneben Nocks/Kärnten. Materialsortierung in Grünschiefer, vielfach Steilstellung. Lärchenwaldregion.

Aufn.. 27. 5. 1953. 


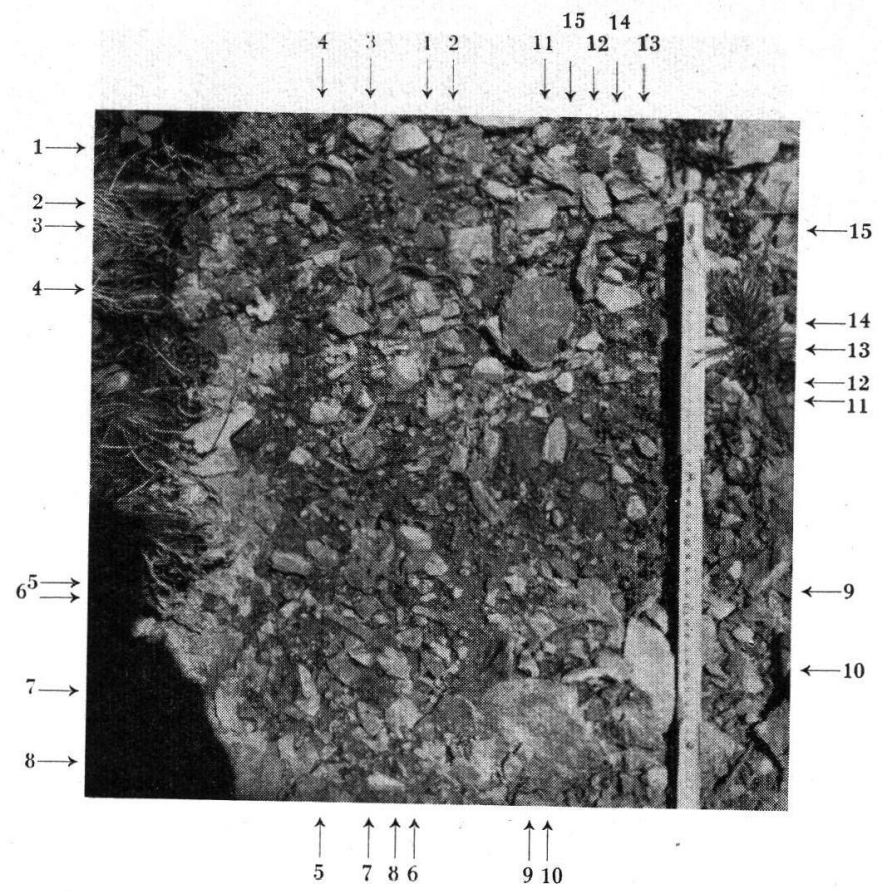

Abb. 3. Frostgeformte Materialsortierungen in $1580 \mathrm{~m}$ Höhe. Ebenheit an der Straße Turracher Höhe - Turrach/Steiermark. Gehobene Lockererde. Verschiedentlich Steilstellung der Steine.
Waldregion. Aufn. 29. 5. 1953.

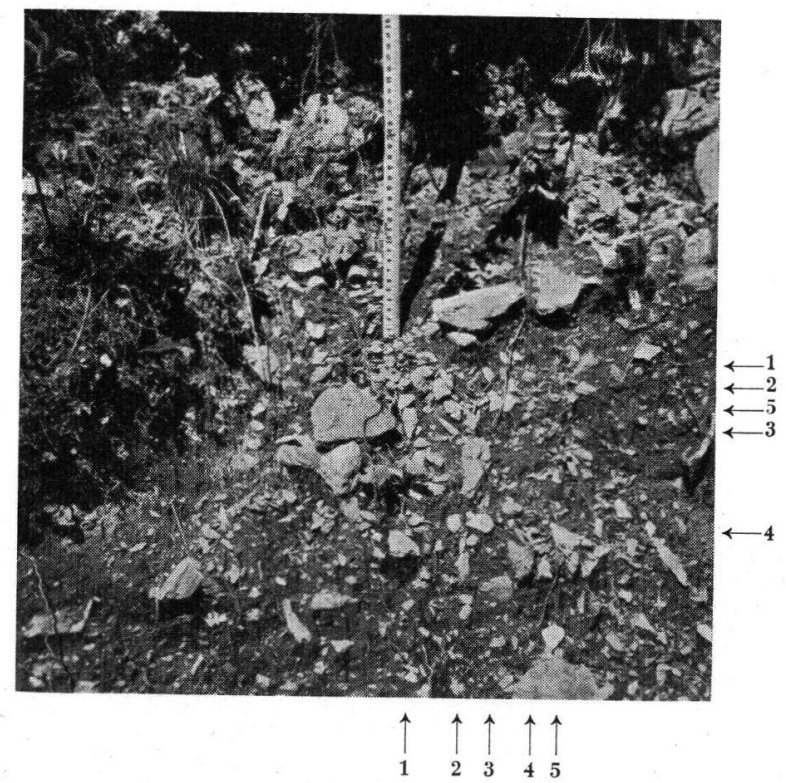

Abb. 4. Initialbildungen frostgeformter Materialsortierung in $1500 \mathrm{~m}$ Höhe am Osthang des Steinturrach/Steiermark. Bergseitige Böschung an der Turracher Straße. - Aufn. 29. 5. 1953. 
in $1740 \mathrm{~m}$ Höhe ausgeprägte Steinnetzwerke vorhanden mit emporgewölbtem Feinerdekern und schräg aufgestellten Steinen. In gleicher Größenordnung, aber in $1700 \mathrm{~m}$ Höhe, unterschied sich ein Steinnetzwerk auf dem Almweg zur Natzalm am Westhang des Schöneben Nocks besonders klar und deutlich von den übrigen (Abb. 2). Der Sortierung unterlagen hier $6-10 \mathrm{~cm}$ große Grünschieferplättchen, die als äußerer Ring immer kleinere Steinchen umschließen (Pfeil 3). Ein Feinerdekern fehlt diesem wie auch den anderen, z. T. gleichgroßen Ringen (Pfeil 1). Gut ausgeprägt sind auch die Steinringe 4, 5 und 6. Die anderen Vorkommen von Steinnetzwerken in diesem Bereich entsprachen in mehreren Beispielen dem von Abb. 2, hatten aber als Kreisinneres ebenso auch Lockererde.

Die Beobachtungen wurden dann von der Turracher Höhe $(1763 \mathrm{~m})$ aus am Osthang des Steinturrach entlang der Straße nach Turrach $(1264 \mathrm{~m})$ weitergeführt. Wie schon betont, kamen nach den besonders tiefen Lagen hin vor allem durch den Straßenbau geschaffene, bergseitige Hangabschnitte als Schuttflächen in verschiedener Neigung in Frage. Je nach den Böschungsverhältnissen bildeten sich kreisförmige oder ovale bis girlandenförmige Steinfiguren heraus. Die Wirkungen der dem Beobachtungstag (29. Mai) vorausgegangenen Frostnacht waren auf allen Schuttflächen deutlich an Hand hochgewölbter Feinerdestellen und Senkrechtstellung des kleinen plattigen Steinmaterials zu sehen. Derartige Feststellungen wurden in 1650, 1625, 1620, 1600, 1580 und $1500 \mathrm{~m}$ gemacht. Als Beispiele davon seien die zwei tiefstgelegenen Vorkommen herausgenommen. In $1580 \mathrm{~m}$ Höhe zeigte eine Schuttebenheit eine Vielzahl von Sortierungsstellen (Abb. 3), von denen auf die der Pfeile 1, 2, 5, 7 und 11-13 hingewiesen sei. Die gleichen Verhältnisse wie bisher waren bei horizontaler und geneigter Schuttfläche in $1500 \mathrm{~m}$ Höhe gegeben. Abb. 4 läßt gleich unterhalb des Maßstabendes einen sehr schönen, bis $2 \mathrm{~cm}$ im Durchmesser großen Steinring erkennen (Pfeil 1), um den herum noch weitere angedeutet sind.

Die Strukturbodenvorkommen am Westhang des Spielriegls $(2167 \mathrm{~m})$ östlich der Turracher Höhe begannen erst in Lagen über $1900 \mathrm{~m}$, boten aber eine ausgesprochene Ubereinstimmung mit den Formen in rund $1700 \mathrm{~m}$. Nahezu in $2000 \mathrm{~m}$ Höhe konnte ein ausgedehnter Frostmusterteppich auf einer Schutthalde in der Nähe einer sehr mächtigen Schneeanwehung beobachtet werden, in dem viele kleine Vegetationsinseln, losgerissen von der geschlossenen Mattendecke, wanderten.

Auf weiteren Begehungen an der Ostseite des Rinsenecks $(2328 \mathrm{~m})$ südwestlich der Turracher Höhe wurden Steinringformen etwa ab $1840 \mathrm{~m}$ in der Nähe der Dichtl-Alm gefunden. Sie waren auf allen vegetationslosen Stellen des Hanges, an welchem ein bedeutender Rasenschlipf im Gange ist, ausgebildet.

Es wurde darauf hingewiesen, daß über und unter der „klimatischen Strukturbodengrenze“ bis $1500 \mathrm{~m}$ herab im Bereiche der Gurktaler Alpen Ansätze zur Strukturbodenbildung vorhanden sind, die sich in Größe und Typus nicht wesentlich voneinander unterscheiden. Sie können als Initialformen mit zum Teil recht klarer Ausbildung aufgefaßt werden. Ihre Häufigkeit auf natürlichen wie auch künstlichen Flächen, ihre Größe und Ausbildung veranlassen uns, sie nicht als außerhalb der bisher angenommenen Strukturbodenregion gelegen aufzufassen, sondern sie in den Gürtel der Strukturbodenvorkommen einzubeziehen. Wenn auch einerseits durch die immer dichter werdende Vegetationsdecke eine Einschränkung der für Strukturbodenbildung geeigneten Flächen stattfindet, so ist dennoch der klimatische Faktor für eine Materialsortierung auch in dieser tiefen Region noch ausgesprochen wirksam. Infolge der tiefen Lage kommt hier ein Typus zum Ausdruck, der dem durch den tageszeitlichen Frostwechselrhythmus bedingten Miniaturtypus von Steinringen und Steinnetzen kleinen und kleinsten Ausmaßes entspricht. So könnte, auf Grund der hier dargelegten Beobachtungen, zu der Feststellung einer zweifachen vertikalen Gliederung von Strukturbodenformen 
noch hinzugefügt werden, daß eine unterste Zone die der Initialformen der Strukturbodenbildung ist, wiederum im Miniaturtypus wie die oberste. Ihre untere Grenze ist in den Gurktaler Alpen bisher bis $1500 \mathrm{~m}$ verfolgt worden.

\section{$\mathrm{Sch}$ rif ten a chwe is}

BAEDEKER, D.: Beiträge zur Morphologie der Gruppe der Schneebergalpen. - Geogr. Jahresber. a. Österr. 12, S. 27. 1922.

BüDEL, J.: Eiszeitliche und rezente Verwitterung und Abtragung im ehemals nicht vereisten Teil Mitteleuropas. - Pet. Mitt. Erg.heft 229, S. 45 ff. 1937. - - Die morphologischen Wirkungen des Eiszeitklimas im gletscherfreien Gebiet. - Geol. Rundschau 34, S. 482-519. 1944.

Conrad, V.: Klimatographie von Kärnten. - Klimatographie von Ơsterreich 6, S. 30 ff. u. 121 ff. 1913. - - Ein Unterwasser-Strukturboden in den Ostalpen. - Gerlands Beiträge 40, S. 353 ff. 1933.

EberLe, G.: Streifenboden am Aufstieg zum Hochvogel. - Natur und Volk 82, S. 1 ff. 1952. Götzinger, G.: Zur Entstehung und Oberflächengestalt der Plateaus der Schnee- und Veitschalm. - Urania 6, S. 171 f. 1913.

HöHL, G.: Beobachtungen über Doppelgrate in den Ostalpen. - Pet. Mitt. 97, S. 174-179. 1953.

KinzL, H.: Beobachtungen über Strukturböden in den Ostalpen. - Pet. Mitt. 74, S. 261-265. 1928.

KLEIN, R.: Klimatographie von Steiermark. - Klimatographie von Österreich 3, S. 55 ff., 63 f. u. 173. 1909.

KrEBs, N.: Klimatisch bedingte Bodenformen in den Alpen. - Geogr. Zeitschr. 31, S. 103. 1925. - - Die Ostalpen und das heutige Ớsterreich 1, S. 96. - Stuttgart 1928.

LEUCHS, K.: Steinringbildung im oberen Lechtal. - Geol. Rundschau 24, 1933.

Matтick, F.: Die Vegetation frostgeformter Böden der Arktis, der Alpen und des Riesengebirges. - Beiträge zur Systematik u. Pflanzengeographie 18, S. 164 ff. 1941.

Mohaupt, W.: Beobachtungen über Bodenversetzungen und Kammeisbildungen aus dem Stubai und dem Grödener Tal. - Diss. Hamburg 1932.

Paschinger, V.: Landeskunde von Kärnten. S. 93 f. - Klagenfurt 1937.

Poser, H.: Das Problem des Strukturbodens. - Geol. Rundschau 24, 1933.

SAlOmon, W.: Arktische Bodenformen in den Alpen. - Sitz.-Ber. Heidelberger Akad. d. Wiss., Math. Nat. K1. 5, S. 1 ff. 1929.

Schadler, J.: Strukturboden (Steinnetze) in der Eislueg, Stodertal, Oberösterreich. - Verhdl. Geol. Bundesanst. Wien 9, S. 205 f. 1931.

SchröDER, P.: Über die vertikale Verteilung der Temperaturschwankungen um den Frostpunkt in Mitteleuropa. - Diss. Leipzig 1912.

Spreitzer, H.: Die Großformung im oberen steirischen Murgebiet. - Festschrift J. Sölch, S. 137 ff. Wien 1951. - - Uber die Entstehung der Großformen der hohen Gurktaler Alpen. Carinthia II, 141. 1951a.

TArnuzzer, Chr.: Beiträge zur Geologie des Unterengadins. - Beitr. z. geol. Karte d. Schweiz, N.F. 23, S. 105 f. 1909. - - Die Schuttfacetten der Alpen und des hohen Nordens. Pet. Mitt. 57, S. 262 ff. 1911.

Troll, C.: Studien zur vergleichenden Geographie der Hochgebirge der Erde. - Bonn 1941. - Die Frostwechselhäufigkeit in den Luft- und Bodenklimaten der Erde. - Meteor. Zeitschr. 60, S. 161 ff. 1943. - - Strukturböden, Solifluktion und Frostklimate der Erde. Geol. Rundschau 34, S. 545-694. 1944. - - Die Formen der Solifluktion und die periglaziale Bodenabtragung. - Erdkunde 1, S. 162-175. 1947. - - Der subnivale oder periglaziale Zyklus der Denudation. - Erdkunde 2, S. 1-21. 1948.

Waldbaur, H.: Schuttglättung und Steinströme im Oberengadin. - Pet. Mitt. 67, S. 195. 1921.

Manuskr. eingeg. 20. 6. 1954.

Anschrift der Verf.: Dr. Gudrun Höhl, Bamberg, Am Zwinger 4 c. 